\title{
NOUVELle
}

\section{Un moteur hybride pour propulser le tapis roulant de l'épithélium intestinal}

Philippe Jay
Institut de génomique fonctionnelle (IGF), université de Montpellier, CNRS, Inserm, Équipe labellisée Ligue contre le cancer, 141 rue de la Cardonille, 34093 Montpellier, France.

philippe.jay@igf.cnrs.fr
> L'épithélium intestinal associe deux fonctions a priori difficiles à concilier. Premièrement, l'absorption des nutriments : à ce titre, il est constitué d'une unique couche de cellules épithéliales non kératinisées, capables d'absorber les nutriments présents dans la lumière intestinale. Mais en même temps, il constitue l'une des principales barrières séparant notre organisme du monde extérieur, reflété dans le contenu de la lumière intestinale, avec des millions de bactéries, virus, parasites, produits toxiques, allergènes, etc. L'intégrité de cette barrière est un enjeu essentiel, et ses défaillances sont associées à de nombreuses maladies locales ou générales, incluant les maladies inflammatoires chroniques de l'intestin [1]. Le renouvellement très rapide, en 4 à 7 jours, de la monocouche des cellules épithéliales de l'intestin permet d'en maintenir l'intégrité malgré son exposition à un environnement en partie hostile.

L'épithélium intestinal est ainsi organisé en deux compartiments, les cryptes et les villosités. Les villosités sont des structures en doigt de gant, se projetant dans la lumière épithéliale et permettant d'augmenter la surface d'absorption de l'épithélium dans l'intestin grêle où la majorité des nutriments sont absorbés. Elles sont essentiellement constituées de cellules spécialisées post-mitotiques. Les cryptes représentent le compartiment prolifératif de l'épithélium intestinal, et contiennent des cellules souches et progénitrices en train de se multiplier qui assurent le renouvellement épithélial. Ainsi, les cellules épithéliales sont produites dans la crypte, puis elles migrent, comme un tapis roulant, vers les villosités, à l'extrémité desquelles elles finissent par se détacher par anoïkis ${ }^{1}$, pour disparaître dans la lumière intestinale. Des travaux pionniers avaient depuis longtemps établi que la production de nouvelles cellules dans l'espace contraint de la crypte intestinale implique la migration du même nombre de cellules vers la villosité [2]. Les résultats de travaux plus récents suggéraient que la prolifération cellulaire dans la crypte constituait la principale force produisant la migration des cellules le long de la villosité [3].

Cette question a récemment connu un rebondissement spectaculaire grâce au travail de Krndija et al., publié dans la revue Science [4]. Arguant du fait que la migration cellulaire n'est pas immédiatement arrêtée après inhibition de la prolifération cellulaire dans les cryptes, par irradiation ou en utilisant des inhibiteurs, les auteurs ont reconsidéré le processus de migration cellulaire le long de l'axe cryptevillosité, en combinant élégamment modèles transgéniques murins, imagerie cellulaire et modélisation mathématique. $\varepsilon n$ effet, la combinaison du marqueur 5-éthynyl-2'-désoxyuridine, incorporé dans les cellules en train de proliférer lors de la phase $\mathrm{S}$ du cycle cellulaire, et d'hydroxyurée (50 mg/kg), un inhibiteur spécifique de la phase $S$, a permis de confirmer que l'inhibition de la prolifération cellulaire ne cause pas un arrêt immédiat de la migration des cellules sur

1 L'anoïkis est une forme particulière d'apoptose, qui est due à un défaut d'interaction entre la cellule et la matrice extracellulaire: lorsque les intégrines de la cellule ne sont plus liées à des protéines de cette matrice, elles transmettent un signal de mort cellulaire. les villosités, suggérant l'existence d'un autre mécanisme de migration. De même, l'observation des densités cellulaires, y compris en présence d'une inhibition de la prolifération, et des vitesses de migration des cellules, à différents niveaux des villosités était compatible avec un modèle théorique combinant des forces motrices issues de la pression de la prolifération cellulaire, mais aussi avec un mécanisme actif de traction des cellules épithéliales. Expérimentalement, la comparaison des forces de tension régnant au sein de l'épithélium intestinal à la base et à l'apex des villosités, dans lesquelles les cellules ne prolifèrent plus, peut donner des indices pertinents sur la prédominance de forces liées à la prolifération cellulaire dans les cryptes, conduisant à un encombrement cellulaire maximal à la base des villosités, ou de forces plutôt liées à une migration active, causant une tension maximale à la base de ces villosités. Des lésions circulaires à la base et à l'apex des villosités ont ainsi permis de mesurer le recul de l'épithélium, dépendant de la tension à laquelle il était soumis, et de montrer l'existence d'une tension à la base des villosités supérieure à celle à leur extrémité, un résultat plutôt en accord avec l'existence d'un processus actif de migration cellulaire.

II restait à identifier le mécanisme tracteur de cette hypothétique migration active. Les cellules migrant activement produisent des forces de traction via des protrusions en lamellipodes ou filopodes, et qui impliquent la polymérisation d'actine ainsi que le complexe Arp2/3, contenant les protéines Arp2 et Arp3 apparentées à l'actine, ainsi que plusieurs 

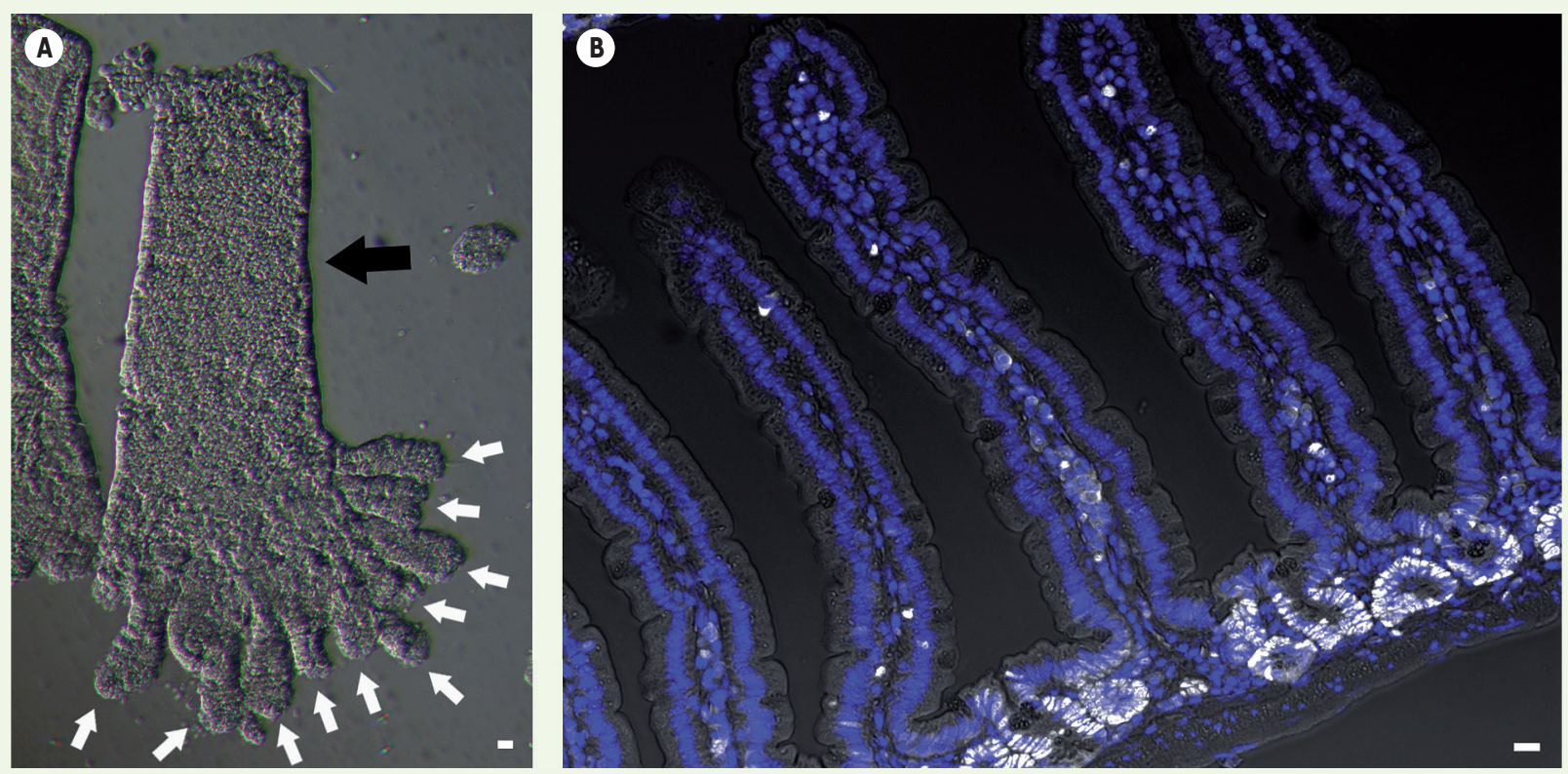

Figure 1. A. Après dissociation de la couche épithéliale du reste de la muqueuse intestinale, on observe, en microscopie à fond clair, des villosités entières (flèche noire), auxquelles restent attachés les cryptes voisines (flèches blanches). Barre d'échelle : $10 \mu \mathrm{mm}$. B. Image en immunofluorescence d'une coupe d'intestin grêle (jéjunum) de souris montrant l'organisation spatiale de l'épithélium intestinal en villosités et en invaginations (cryptes de Lieberkühn). Les noyaux cellulaires sont marqués par le DAPI (en bleu) et les cellules des cryptes en train de se multiplier sont marquées grâce à un anticorps anti-Ki67 (en blanc). On visualise ainsi clairement la démarcation entre la crypte, compartiment où les cellules prolifèrent, et la villosité, compartiment différencié. Barre d'échelle : $10 \mu \mathrm{m}$ (Photos @ François Gerbe).

autres sous-unités [5]. Cependant, de telles structures n'avaient pas encore été décrites dans les cellules épithéliales intestinales. Les auteurs ont utilisé deux systèmes rapporteurs in vivo. Le système LifeAct-mCherry permet de visualiser les régions riches en filaments d'actine, tandis qu'une autre lignée de souris a été modifiée pour permettre l'expression de la protéine fluorescente GFP (green fluorescent protein) à la membrane cellulaire. C'est ainsi qu'ils ont réussi à mettre en évidence des protrusions basales des entérocytes, en contact avec la membrane basale et pointant en direction de la migration cellulaire (Figure 1). De plus, l'interférence avec la fonction de Arp2/3, par l'utilisation d'inhibiteurs spécifiques (CK-666) ou via une déficience génétique (souris Arpc4fl/fl; Villin-CreERT ${ }^{2}$ ), a conduit à une perte de la polarité « avant-arrière » des protrusions et à un arrêt de la migration des cellules sur les villosités, sans affecter la prolifération cellulaire dans les cryptes ni les jonc- tions intercellulaires. Enfin, l'analyse de la tension cellulaire sur les villosités, en combinaison avec des traitement à l'hydroxyurée (1 $250 \mathrm{mg} / \mathrm{kg}$ ) ou avec l'inhibiteur de Arp2/3, a permis de confirmer la nature hybride des forces motrices de la migration des cellules épithéliales intestinales : combinaison d'une force liée à la prolifération cellulaire dans les cryptes et d'une force de traction cellulaire dépendante d'Arp2/3.

Alors que le travail de Krndija et al. révèle que la migration des cellules épithéliales le long de l'axe crypte-villosité est en partie active, d'autres questions demeurent sans réponse. Par exemple, la jonction entre la crypte et la villosité possède des caractéristiques uniques dans l'axe crypte-villosité. En effet, des colonnes de cellules issues de cryptes distinctes se rejoignent sur une même villosité, compliquant l'interprétation des densités et tensions cellulaires entre base et apex de la villosité. Par ailleurs, les signaux permettant de guider la migra- tion cellulaire unidirectionnelle vers le sommet des villosités restent à découvrir; la pression mitotique du «côté crypte » des cellules épithéliales joue-telle un rôle dans l'orientation des protrusions cellulaires riches en actine? Quel est le rôle de signaux impliquant les récepteurs Eph des éphrines, responsables de la localisation des cellules de Paneth $^{2}$ au fond des cryptes [6], dans l'orientation de la migration cellulaire vers l'extrémité des villosités? Finalement, il sera intéressant d'interroger la pertinence de la découverte de propriétés migratoires des cellules épithéliales intestinales dans le contexte tumoral, notamment au front d'invasion, ouvrant ainsi la voie à de nouvelles pistes thérapeutiques. $\diamond$

Hybrid propulsion for the intestinal epithelium conveyor belt

\footnotetext{
2 Les cellules de Paneth élaborent des grains de sécrétion contenant du lysozyme, enzyme capable de détruire la paroi bactérienne. Leur cytoplasme est riche en lysosomes.
} 


\section{LIENS D'INTÉRÊT}

L'auteur déclare n'avoir aucun lien d'intérêt concernant les données publiées dans cet article.

\section{RÉFÉRENCES}

1. César Machado MC, Silva FP da. Intestinal barrie dysfunction in human pathology and aging. Curr Pharm Des 2016 ; 22 : 4645-50.

2. Cheng $H$, Leblond CP. Origin, differentiation and renewal of the four main epithelial cell types in the mouse small intestine. I. Columnar cell. Am J Anat $1974 ; 141: 461-79$.

3. Parker A, Maclaren 0J, Fletcher AG, et al. Cell proliferation within small intestinal crypts is the principal driving force for cell migration on villi. FASEB J 2017 ; $31: 636-49$.

4. Krndija D, Marjou FE, Guirao B, et al. Active cel migration is critical for steady-state epithelial turnover in the gut. Science 2019 ; 365 : 705-10.

5. Buracco S, Claydon S, Insall R. Control of actin dynamics during cell motility. F1000Res 2019 ; 8 : doi: 10.12688/f1000research.18669.1.
6. Batlle $\varepsilon$, Henderson JT, Beghtel H, et al. Beta-catenin and TCF mediate cell positioning in the intestina epithelium by controlling the expression of $\mathrm{EphB} /$ ephrinB. Cell 2002; 111 : 251-63.

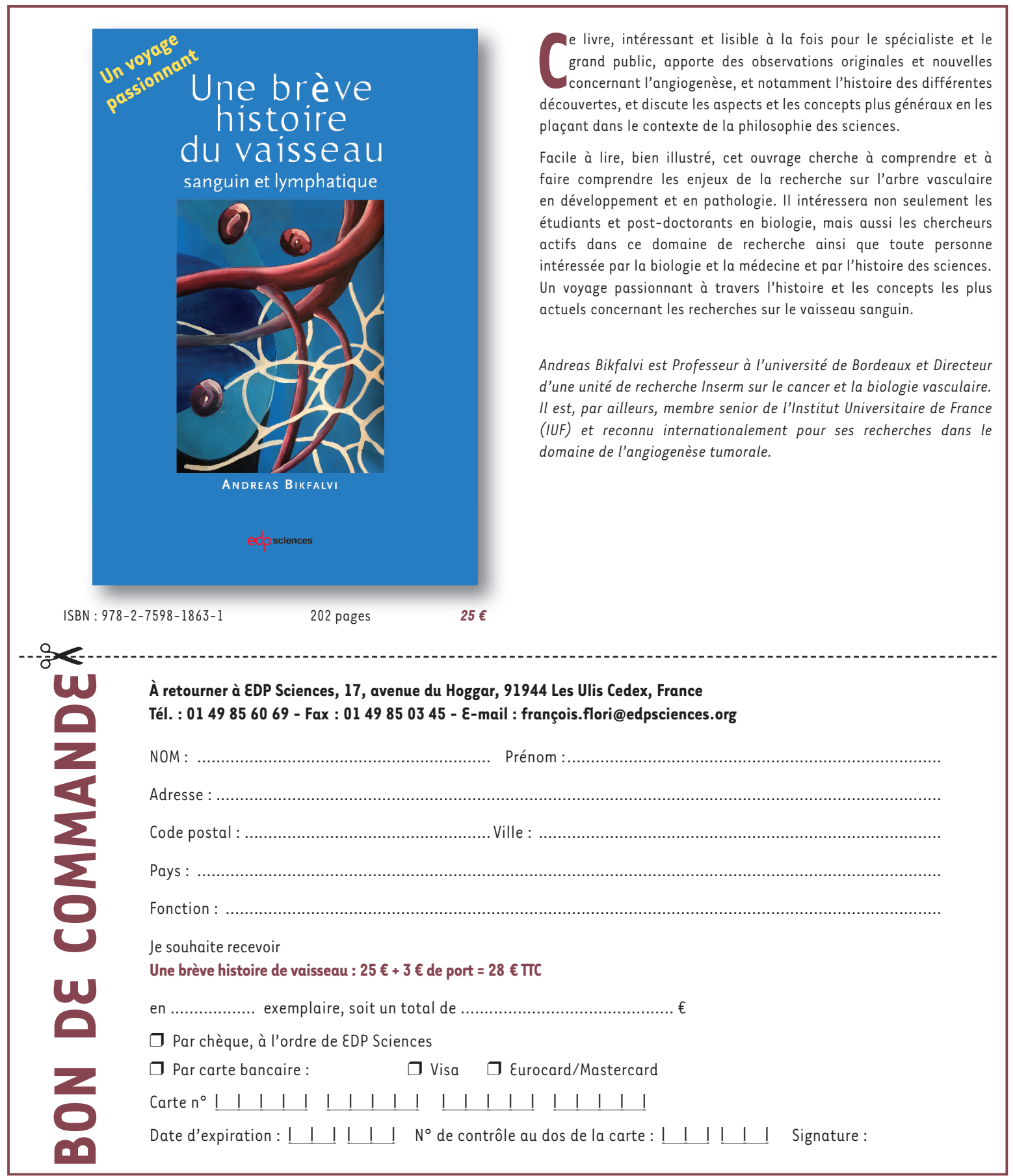

